\title{
Écriture réflexive et réflexion critique dans l'exercice du compte rendu
}

\author{
Serge Bibauw
}

\section{(2) OpenEdition}

1 Journals

Édition électronique

URL : http://journals.openedition.org/ripes/358

DOI : 10.4000/ripes.358

ISSN : 2076-8427

Éditeur

Association internationale de pédagogie universitaire

Référence électronique

Serge Bibauw, «Écriture réflexive et réflexion critique dans l'exercice du compte rendu », Revue internationale de pédagogie de l'enseignement supérieur [En ligne], 26(1) | 2010, mis en ligne le 10 mai 2010, consulté le 10 décembre 2020. URL : http://journals.openedition.org/ripes/358 ; DOI : https:// doi.org/10.4000/ripes.358

Ce document a été généré automatiquement le 10 décembre 2020.

Article L.111-1 du Code de la propriété intellectuelle. 


\title{
Écriture réflexive et réflexion critique dans l'exercice du compte rendu
}

\author{
Serge Bibauw
}

\section{NOTE DE L'AUTEUR}

Ce texte applique les recommandations orthographiques du Conseil supérieur de la langue française.

\section{Contexte}

\section{1. Écriture réflexive et développement autonome des étudiants}

1 L'étudiant entamant des études supérieures fait face à une série de nouveaux défis et de nouvelles exigences quant à sa façon d'apprendre et de s'impliquer dans sa formation. Un élément déterminant dans la réussite de sa formation sera donc sa capacité à s'adapter à ce nouveau cadre. Il semble par ailleurs aujourd'hui de plus en plus essentiel de former des étudiants capables de poursuivre de façon autonome leur développement, au-delà de la transmission directe de savoirs et de savoir-faire institués. Dans cette perspective, la réflexivité - à savoir, la capacité de retour sur soi apparait comme une compétence transversale centrale à développer, en tant que capacité métacognitive de retour de l'apprenant sur sa façon d'apprendre (Romainville, 2007), mais aussi, de façon plus large, en tant que capacité de distanciation et de retour sur son cheminement personnel et ses pratiques (Bibauw et Dufays, à paraitre).

2 Pour chercher à développer cette compétence, de nombreux enseignants-chercheurs ont mis en avant l'écriture réflexive comme source de développement du sujet (Chabanne, 2006 ; Dufays et Thyrion, 2004 ; Scheepers, 2006). Qu'entend-on par écriture 
réflexive ? Le recours à cette idée s'est fait dans la littérature scientifique dans deux traditions distinctes, qui impliquent deux approches différentes de l'objet : d'un côté, des formateurs d'enseignants se revendiquant de Schön (1994) qui cherchent à développer la réflexivité chez les futurs enseignants (Paquay et Sirota, 2001); de l'autre, des didacticiens spécialisés dans les pratiques langagières, se référant au socioconstructivisme et à Vygotski (1997), qui analysent la distanciation et le rapport à l'écrit des élèves (Chabanne et Bucheton, 2002). Le regard porté sur l'écriture réflexive diffère alors nettement: dans la tradition schönienne, celle-ci est une activité spécifique d'écriture, à travers lesquelles l'apprenant effectue explicitement un retour sur son action professionnelle; dans la tradition vygotskienne, on analyse plutôt des pratiques d'écriture existantes, de toutes sortes, pour évaluer la distanciation cognitive que l'écriture engendre chez l'apprenant (à quel point cette écriture est « réflexive ») sans donc que ces pratiques soient définies par un objet scriptural caractéristique (Bibauw et Dufays, à paraitre).

Dans le cadre d'un projet de recherche FNRS-FRFC ${ }^{1}$, nous nous sommes interrogés sur la valeur des pratiques d'écriture réflexive pour les étudiants entrant à l'université. L'objectif était en particulier de savoir quelles formes d'écrit réflexif étaient pratiquées et quels en étaient les effets sur la motivation et le sens des apprentissages de ces étudiants.

4 Puisqu'il s'agissait de porter notre regard sur une série de pratiques spécifiques, nous avons privilégié l'approche schönienne de la réflexivité, selon laquelle celle-ci est perçue comme une activité mentale spécifique. Dans cette perspective, l'écriture réflexive se présente sous un certain nombre de formes discursives : rapport de stage (Dufays, 2004), mémoire professionnel (Crinon et Guigue, 2002), carnet ou journal de bord (Bishop et Doquet-Lacoste, 2008; Scheepers, 2004), portfolio (Ricard-Fersing, Dubant-Birglin et Crinon, 2002 ; Vanhulle, 2006) ... Ces formes d'écriture réflexive ont été largement développées dans le cadre des formations professionnalisantes (notamment en formation des enseignants), mais se sont également étendues aux formations de type général, notamment au premier cycle universitaire, par exemple à travers le développement de journaux et portfolios d'apprentissage (Cerisier, 2006; Crinon, 2002). Le portfolio, en particulier, s'est au cours des dernières années imposé comme un dispositif majeur d'encadrement de l'autoapprentissage des étudiants (Gauthier, 2008).

\subsection{Quelles pratiques d'écriture réflexive à l'entrée à l'université ?}

5 Dans un premier temps, nous avons cherché à identifier une diversité de pratiques d'écriture réflexive réalisées en première année d'université. Nous nous sommes alors heurtés aux premiers obstacles qui font qu'un recensement des pratiques réflexives effectives n'a jamais été réalisé jusqu'à présent ${ }^{2}$.

Le problème est que l'objet "pratique réflexive ", que ce soit au sens schönien ou au sens vygotskien, n'est pas un objet institué, constitué par des frontières définies : toute pratique est d'une certaine façon susceptible d'être évaluée quant à son niveau de réflexivité (interne ou induite). Chez les chercheurs, le concept de réflexivité apparait ainsi plus comme un concept-étiquette, utilisé pour décrire une pratique déterminée, qu'un concept-catégorie, qui permettrait de segmenter le champ des pratiques réelles en 
établissant des critères discriminatoires de ce qu'est une pratique réflexive. Dès lors, où doit-on s'arrêter lorsqu'on cherche à recenser les pratiques réflexives?

7 Le deuxième obstacle vient $\mathrm{du}$ fait que très peu d'enseignants - que ce soit dans l'enseignement secondaire ou supérieur - utilisent spontanément le concept de réflexivité pour décrire leurs démarches pédagogiques.

De ce fait, soit l'on considère, comme Chabanne et Bucheton (2002), Scheepers (2010) et d'autres chercheurs se réclamant de l'approche vygotskienne, que le langage est « intrinsèquement réflexif », auquel cas toute pratique faisant intervenir les capacités de production langagière des étudiants est susceptible d'être analysée; soit on se résigne à ne considérer que certaines formes-types de pratique réflexive déjà connues, comme le portfolio, le journal de formation, le rapport de stage... et à étudier leur utilisation dans les programmes de formation visés. Étant donné l'impossibilité de prendre en compte la totalité des pratiques langagières réalisées par les étudiants du supérieur, nous avons plutôt opté pour cette deuxième option.

D'un point de vue global, notre enquête au sein des programmes de 1re année d'université n'a pas fait apparaitre de tendances fortes à l'utilisation de l'écriture réflexive. Celle-ci se voit souvent cantonnée, comme on pouvait l'imaginer, à des formations professionnalisantes (où elle vient notamment encadrer les va-et-vient entre théorie et pratique) - donc plutôt dans les cycles courts non universitaires ou en 2e cycle, pour les filières professionnalisantes. Cependant, il y a quelques lieux où des démarches pédagogiques innovantes ont recours à l'écriture réflexive entendue comme retour sur une pratique. Dans plusieurs cas, celle-ci intervient dans une dynamique de tâche-projet où l'étudiant, après avoir réalisé un travail de groupe relativement conséquent, est appelé à s'interroger sur la façon dont s'est déroulé le travail, ce qu'il en a appris et/ou ce que ça lui a enseigné sur son choix d'orientation professionnelle. De telles pratiques ont pu être constatées notamment dans les activités de 1re année en sciences, en sciences de l'ingénieur et en psychologie.

Dans le cadre de cet article, afin de mieux évaluer quel était l'impact de l'écriture réflexive sur les étudiants, nous nous sommes penchés sur une pratique particulière, très récemment développée en philosophie et lettres, à savoir un dispositif de portfolio étudiant appelant à réaliser des comptes rendus d'activités extraacadémiques.

\subsection{Un ePortfolio pour valoriser les activités extraacadémiques des étudiants de première année en histoire de l'art et archéologie}

11 À la rentrée 2008-2009, a été mis en place à l'Université catholique de Louvain un dispositif de portfolio électronique, ou "ePortfolio ", intégré dans les trois années du baccalauréat en histoire de l'art et archéologie ${ }^{3}$. Cette expérience d'ePortfolio vise principalement à valoriser la réalisation par les étudiants d'activités extraacadémiques entrant dans le cadre de leur formation (p. ex. visites de musées, d'expositions et de sites du patrimoine, assistance à des conférences et des colloques...), permettant d'inclure dans le cycle d'études une dimension d'autoformation de l'étudiant qui soit néanmoins encadrée et évaluée en fonction du cadre universitaire (Druart, Verslype et Corten-Gualtieri, 2009). À cette fin, un ensemble de consignes et de moments de suivi individuel ont été prévus dans le dispositif. 

son amplitude. Il nous importe de souligner néanmoins la qualité et la finesse du travail de conception réalisé par l'équipe pédagogique responsable de ce projet : le dispositif se présente comme ambitieux et très complet et permet de répondre avec brio aux défis que représentent le suivi individuel et régulier d'un grand nombre d'étudiants et la gestion d'une grande variété d'activités laissées au libre choix de l'étudiant. Pour plus d'informations, on se réfèrera à l'article de Druart et al. (2009), qui décrit en détail le fonctionnement global du dispositif.

Concrètement, l'ePortfolio se matérialise principalement en la réalisation individuelle par l'étudiant d'une série de comptes rendus d'activités extraacadémiques: après chaque activité, l'étudiant doit remplir un formulaire de plusieurs pages et le transmettre au formateur. Ce compte rendu a un double rôle : d'une part, attester de la réalisation de l'activité par l'étudiant (en demandant de compléter différentes informations factuelles et éventuellement de remettre une attestation) et d'autre part, « encadrer l'autonomie » de l'étudiant en le poussant à porter un regard plus spécialisé sur les faits culturels (Druart et al., 2009, p. 23). Encadrer l'autonomie, cela passe à la fois par la sollicitation d'un effort d'analyse approfondie et critique de l'activité, « qui réponde aux exigences universitaires », et par "l'induction d'une posture réflexive » chez l'étudiant (23). Au-delà de la complétion de formalités académiques, chaque compte rendu requiert donc de l'étudiant un travail de rédaction important à travers quatre rubriques à remplir :

1. « objectifs et motivations de l'étudiant»;

2. «compte rendu de l'activité", à savoir une description factuelle et objectivante de l'objet culturel observé, ou d'une partie (p. ex. une salle d'un musée) ;

3. « regard critique sur l'activité »;

4. « autoévaluation des apprentissages liés à la participation à l'activité ».

Il importe de souligner que des consignes précises sur ce qui était attendu dans chacune de ces rubriques sont annexées sous forme de grille d'évaluation, les attendus pouvant varier en fonction du type d'activité. Ainsi est-il précisé, pour la rubrique «regard critique» d'une visite de musée, que l'étudiant doit avoir produit une «réflexion argumentée concernant la pertinence dans le choix des œuvres [...]» et avoir porté " un regard critique sur l' "efficacité" (ou non) de la scénographie [...] $»^{4}$. Pour la rubrique "autoévaluation des apprentissages », il est demandé que "l'étudiant relate ce que l'activité lui a apporté (ou pas) en termes de savoirs » d'une part, et «d'attitudes [...] et de valeurs » d'autre part.

Notre étude a été réalisée au cours de la première année de fonctionnement de cette expérience, donc exclusivement sur des étudiants de première année, qui devaient réaliser chacun quatre comptes rendus. Notre objet de recherche étant les aspects réflexifs de l'écriture du compte rendu, nous n'avons étudié que la partie rédigée de ces productions (nous n'analyserons donc pas l'ensemble du dispositif), et plus particulièrement les rubriques "regard critique» et "autoévaluation des apprentissages ». Ces deux rubriques nous semblaient en effet particulièrement intéressantes dans la mesure où elles invitent l'étudiant à une certaine prise de distance critique, mais à travers deux postures d'écriture à priori très différentes. Nous reviendrons sur ces similitudes et ces différences ci-dessous. 


\section{Méthodologie}

16 Il nous faut d'abord préciser que nous ne sommes et n'avons été en rien impliqués dans le projet ePortfolio - ni dans son élaboration, ni dans son suivi, ni dans le suivi et l'évaluation des étudiants. Toutes nos mesures et nos observations ont été réalisées à postériori, sans concertation avec l'équipe pédagogique derrière le projet.

Notre première question de recherche était double : comment les étudiants mettent-ils en œuvre l'écriture réflexive et comment la perçoivent-ils? En étudiant le dispositif d'ePortfolio, nous avons donc analysé dans un premier temps la façon dont les étudiants remplissaient les rubriques "regard critique " et "autoévaluation des apprentissages ", avec l'objectif d'y déceler une posture discursive différente, liée au type de distanciation demandé (implicitement) par les consignes.

Nous avons ainsi analysé les portfolios de tous les étudiants de première année ayant participé à l'expérience et n'ayant pas abandonné leur projet d'études en cours d'année ${ }^{5}$, soit 58 étudiants. La moyenne des résultats obtenus en dernière session par ces étudiants était de 54,1\% (écart type : 14,4).

Nous avons analysé les productions des élèves à la fois d'un point de vue qualitatif, à l'aide d'outils d'analyse discursive, et d'un point de vue plus quantitatif, plus mesurable. Les analyses qualitatives seront relativement peu détaillées ici, dans la mesure où elles ne se sont pas toutes révélées très instructives pour notre question de recherche.

Les analyses quantitatives visaient quant à elle à chercher à comprendre les critères implicites de l'évaluation des portfolios par les formateurs (cf. infra) en les mettant en rapport avec le contenu des portfolios. Nous avons réalisé trois analyses quantitatives élémentaires : longueur (nombre de mots), respect des normes langagières (nombre de fautes d'orthographe, de syntaxe et de ponctuation par rapport au nombre de mots) et présence du locuteur (occurrences de pronoms personnels de la 1re personne, par rapport au nombre total de mots). Étant donné l'amplitude des données, mais aussi du fait que ces analyses quantitatives sont relativement annexes au sein de notre projet de recherche, nous avons mené ces analyses de façon semi-automatisée à l'aide du logiciel de vérification orthographique Antidote. Le comptage des fautes de norme et le calcul de la présence des locuteurs ne sont donc réalisés qu'à titre indicatif et relatif, et pourraient être affinés dans une étude ultérieure. En particulier, l'évaluation de la présence du locuteur par le simple comptage des pronoms personnels de la première personne est, bien sûr, une pratique relativement simpliste.

21 Outre l'analyse textuelle des comptes rendus, nous avons tenu compte des commentaires d'évaluation des formateurs pour chaque compte rendu. En particulier, nous avons principalement considéré l'évaluation du premier compte rendu, dans la mesure où l'activité analysée était la même pour tous les étudiants («activitétremplin » imposée à titre strictement formatif), où les évaluateurs pour cette activité n'étaient que deux (contrairement aux huit formateurs qui suivaient par la suite les étudiants) et où une notation chiffrée (sur 5) était donnée à titre indicatif au compte rendu.

Ensuite, afin de recueillir un retour direct sur la mise en œuvre et la perception des comptes rendus par les étudiants, nous avons réalisé des entretiens de type compréhensif (Kaufman, 2004) tantôt individuels, tantôt avec des duos d'étudiants, afin 
d'obtenir des retours les plus personnels possible sur l'expérience vécue par les étudiants. Au fond, il s'agissait pour nous, en entretien et oralement, de demander aux étudiants une "autoévaluation des apprentissages» - au sens large - concernant la totalité de l'expérience de l'ePortfolio. Ces entretiens semi-dirigés se sont révélés très instructifs, en particulier quand ils étaient menés avec des duos d'étudiants, dans la mesure où ils donnaient lieu ainsi à des échanges coconstruits entre étudiants et à des réflexions plus approfondies.

Ces entretiens ayant été réalisés sur une base strictement volontaire par les étudiants, tous n'y ont pas participé : nous avons interrogé 14 étudiants. Cet échantillon étudié plus en détail à travers les entretiens compréhensifs représentait donc $24 \%$ de la population cible. L'échantillon est relativement représentatif du groupe entier, dans la mesure où tant des étudiants faibles (redoublant pour certains leur première année) que forts se sont proposés. La moyenne des résultats est néanmoins légèrement supérieure à celle du groupe entier : 59,3\% (écart type : 11,1); cependant, cette différence n'est pas significative $(z=1,19)$. La moyenne de cet échantillon correspond presque exactement à la moyenne minimale exigée pour considérer l'année réussie (60\%), ce qui nous permet de supposer que cet échantillon correspond assez bien au niveau moyen attendu en première année d'université.

Nos analyses ont réellement été prioritairement construites au départ des témoignages étudiants récoltés dans les entretiens, de façon inductive donc, sur les principes de lathéorie ancrée, ou grounded theory (Barney et Strauss, 1967). Le recours aux analyses quantitatives n'a eu lieu que de façon annexe et postérieure, pour valider des hypothèses secondaires. Pour cette raison, nous avons souhaité présenter différents extraits de corpus pour appuyer nos observations. Dans ces extraits, les locuteurs sont identifiés par un code de corpus en minuscules (upb) et un code de locuteur en majuscules (CR1 p.ex. ; le code E0désigne le chercheur qui mène l'entretien).

\section{Résultats}

\subsection{Réception du dispositif global}

\subsubsection{Engagement et motivation intrinsèque}

Il importe de signaler que nos enquêtes ont révélé une très bonne réception du dispositif par les étudiants. Les responsables du projet pédagogique ont pu observer un investissement important des étudiants et une mise en œuvre dans l'ensemble très réussie du dispositif au cours de sa première année d'application. Les étudiants que nous avons interrogés en entretien compréhensif se sont tous présentés comme pleinement engagés dans l'expérience.

Tous les étudiants interrogés ont perçu dans leurs retours des apports positifs de l'expérience. Ils ont souligné deux aspects essentiels ayant influé positivement sur leur motivation intrinsèque pour le projet. D'abord, la liberté laissée dans le choix des activités est très nettement l'élément le plus encourageant du dispositif ; elle développe en effet la perception de contrôlabilité de l'apprenant, qui intervient directement sur sa motivation (Viau et Bouchard, 2000). Ensuite, le suivi personnalisé des étudiants par les formateurs, à travers les corrections commentées des comptes rendus et les entretiens individuels annuels, est le second apport majeur du dispositif, dans la 
mesure où il donne aux étudiants une possibilité d'ajustement individuel qui pourra améliorer leur sentiment de compétence. Par ailleurs, le dispositif a également été évalué de façon positive par les étudiants parce qu'il les poussait à s'ouvrir à la vie culturelle et à réaliser des activités qu'ils n'auraient peut-être pas faites sans cette incitation, et parce qu'il introduisait un type de travail différent et original au sein de leur formation universitaire.

Dans l'ensemble, la quasi-totalité des étudiants interrogés estime le bilan motivationnel positif : la valeur de la tâche est perçue comme supérieure à l'investissement cognitif et temporel qu'elle exige. Seule une étudiante a fait part d'un bilan plus mitigé et d'une dynamique de relatif désengagement, en insistant sur le fait que l'investissement en temps nécessaire en première année, conjugué à l'importance des travaux d'autres cours, était difficilement gérable. Il s'agit cependant d'une étudiante qui présente manifestement des lacunes importantes et une posture peu adéquate par rapport aux attentes de l'enseignement supérieur. Ceci met néanmoins en évidence le fait qu'une pratique pédagogique comme l'ePortfolio, misant sur l'apprentissage en autonomie, n'est clairement pas un secours pour les étudiants présentant un décalage majeur avec les prérequis universitaires.

\subsubsection{Trois temps dans la perception du dispositif}

Les entretiens ont révélé le fait que la perception par les étudiants du dispositif d'ePortfolio évolue en trois temps, au fur et à mesure de son assimilation.

Lors de la présentation du projet pédagogique, les étudiants ont globalement reçu l'idée avec enthousiasme: la perspective de découverte (visites de patrimoine, activités extraacadémiques, libre choix de l'activité) en particulier a été perçue comme attractive.

Dans un second temps, après avoir assisté à la première activité - commune et imposée - et avoir produit le compte rendu, cet enthousiasme a fait place à un certain découragement face à l'importance du travail de rédaction attendu pour le compte rendu. L'impression est alors que l'intérêt et la découverte ne compensent pas la lourdeur du dispositif. Il est néanmoins clair ici que le caractère imposé de cette première activité entre en jeu dans cette perte de motivation.

31 Cependant, tous les étudiants (à l'exception de l'étudiante que nous avons évoquée précédemment) font part ensuite d'un regain d'intérêt pour l'ePortfolio après la réalisation du deuxième compte rendu, au départ d'une activité choisie. Il semble qu'ils perçoivent alors finalement l'intérêt de réaliser un compte rendu extensif, critique et réflexif au départ de l'activité vécue.

\subsubsection{Assimilation des exigences universitaires}

Ce troisième temps, dans lequel les étudiants estiment que l'expérience est intéressante et utile pour leur formation, nous semble devoir être compris à la fois comme une prise de conscience de la valeur d'apprentissage du dispositif et comme une assimilation progressive des attentes de l'enseignement universitaire par les étudiants. Leurs témoignages montrent en effet une sorte d'acceptation du dispositif du fait de la légitimité de certaines exigences dans le cadre académique. Ceci se marque notamment à travers des tournures impersonnelles (« oui, c'est vraiment important [pour notre 
formation] ») dans lesquelles ils soulignent l'importance de développer un «regard critique », de « tout justifier », de " creuser les choses » ...

Manifestement, au cours de leur première année de cours, les étudiants assimilent donc petit à petit les contraintes du dispositif, associée à une intériorisation progressive des attentes et des critères de validité académiques. Progressivement, dans un processus d'acculturation, ils font leur le discours universitaire sur les exigences critiques légitimes de l'université. Ainsi, cette assimilation de « ce qu'il est normal qu'on attende de moi dans le cadre universitaire » concourt à l'acceptation du dispositif.

\subsection{Mise en œuvre des comptes rendus, à travers deux rubriques- clés}

\subsubsection{Regard critique et autoévaluation des apprentissages : deux postures particulières}

Deux rubriques des comptes rendus nous semblent particulièrement intéressantes: « regard critique » et "autoévaluation des apprentissages». Il s'agit en effet dans les deux cas pour l'étudiant de porter un regard distancié, plus profond (ces rubriques suivent la description factuelle de l'activité, dans lequel une approche relativement primaire de l'objet est demandée), sur l'activité réalisée. En ce sens, il s'agit dans les deux cas pour l'étudiant de faire preuve de réflexion critique rétrospective.

Cependant, l'objet sur lequel porte le regard critique est différent : extérieur à soi pour la première (par exemple, critiquer la scénographie d'une exposition), autocentré pour la seconde (on doit expliciter ses propres apprentissages). De cette distinction d'objet découle une distinction de posture énonciative attendue dans la rédaction de chaque rubrique. Pour le regard critique, il est attendu de l'étudiant une énonciation objectivante et validante, semblable à celle valorisée pour la plupart des productions discursives étudiantes à l'université (Crahay et Marlair, 2008). À l'inverse, l'autoévaluation des apprentissages exige plutôt de l'étudiant qu'il se mette dans une posture d'écriture peu commune dans le cadre scolaire : une posture autocentrée, quasi autobiographique, et à la fois profondément heuristique. Nous définirons par ces caractéristiques ce que la plupart des études désignent comme une "posture réflexive » (Girardeau et Huver, 2009).

Ces deux rubriques présentent donc l'intérêt de mettre en parallèle, d'un côté, une écriture critique plus conventionnelle, hétérocentrée et objectivante, et de l'autre, une écriture proprement réflexive, autocentrée et subjectivante. Dans le cadre de notre étude, nous chercherons donc à savoir comment les étudiants assimilent cette diversité de postures énonciatives dans leurs comptes rendus et quels sont les apports et les spécificités éventuels de l'écriture réflexive par rapport à l'écriture critique.

\subsubsection{Difficultés de mise en œuvre de l'autoévaluation des apprentissages}

Il ressort tout d'abord des entretiens avec les étudiants que l'autoévaluation des apprentissages est la rubrique présentant la plus grande difficulté pour eux. C'est une observation unanime et saillante chez tous les étudiants. Cependant, pour la plupart, cette difficulté n'est pas ressentie en termes de complexité ou d'exigence, mais résulte plutôt d'une incompréhension des attentes des évaluateurs et d'un sentiment d'incapacité à y répondre. 
Cte incompréhension est sans doute à imputer partiellement à la nouveauté du genre discursif pour les étudiants, qui ont tous le sentiment d'avoir affaire à une pratique qu'ils n'avaient jamais exercée auparavant. Sans doute des consignes plus détaillées encore et un exercice préalable spécifique à ce type de rédaction eussent-ils pu les aider quelque peu. Néanmoins, au-delà de la nouveauté du genre, on peut se demander dans quelle mesure le cadre dans lequel l'activité réflexive est demandée n'est pas également responsable du relatif blocage rencontré par les étudiants.

\begin{tabular}{|l|l|}
\hline upbKM1 & $\begin{array}{l}\text { euh le savoir, ça ça pouvait aller / vu que c'est plutôt ce qu'on en a appris au niveau } \\
\text { théorique / mais le savoir-être } \\
\text { rubrique }\end{array}$ \\
\hline upbE0 comprenais pas trop / ce qu'il fallait mettre dans la \\
\hline upbKM1 & $\begin{array}{l}\text { ok // et tu as petit à petit mieux compris ou pas trop? } \\
\text { souvent ça euh / pas assez de savoir-être ou alors pas du tout / et puis je me forçais à en } \\
\text { trouver et en y réfléchissant bien oui il y en avait quelques-uns mais je dois dire que } \\
\text { c'étaient souvent les mêmes }\end{array}$ \\
\hline
\end{tabular}

Les témoignages d'étudiants font ainsi état de différentes démarches et stratégies réalisées pour dépasser la difficulté rencontrée. Ces démarches sont tantôt intrinsèques - «se forcer à trouver » (voir ci-dessus), « chercher très loin pour essayer de remplir les caractères", revenir aux consignes, avancer par essai/erreur en s'aidant des commentaires d'évaluation ou de l'accompagnement du formateur -, tantôt extrinsèques - demander des idées à un proche (parents en général), espérer/souhaiter que le formateur accorde moins d'importance à cette rubrique -, mais dans l'ensemble, comme notre énumération le laisse apparaitre, ces démarches de résolution correspondent plus à des stratégies d'évitement de l'activité réflexive qu'à l'intégration progressive d'une posture réflexive. Il semble donc que le cadre dans lequel est appelée la démarche réflexive ne soit pas vraiment propice à son développement, encourageant plutôt des stratégies d'évitement.

\subsubsection{Perception de l'importance et de l'intérêt des rubriques}

Les entretiens ont par ailleurs fait apparaitre une nette différence dans la perception subjective que les étudiants ont de la rubrique « regard critique " par rapport à celle d'« autoévaluation des apprentissages ». Naturellement, les étudiants ont tendance, dans leurs témoignages, à mettre en parallèle ces deux rubriques en soulignant l'importance du regard critique, en termes de valeur, d'utilité et de correspondance aux critères de validité académique, par rapport à l'autoévaluation des apprentissages, globalement considérée comme secondaire.

\begin{tabular}{|l|l|} 
upbDS1 & $\begin{array}{l}\text { moi personnellement je pense que ça change pas grand-chose / je pense que le point le } \\
\text { plus important c'est le regard critique / et euh / enfin pour moi / et euh le savoir-être } \\
\text { savoir-faire/ enfin savoir savoir-être, moi je vois pas trop euh / enfin ouais / je vois pas } \\
\text { trop l'intérêt de ce cadre/ de cette question-là }\end{array}$
\end{tabular}

Revue internationale de pédagogie de l'enseignement supérieur, 26(1) | 2010 
upbCL1 ou alors qu'ils le mettent mais qu'ils y accordent moins d'importance parce que/

41 Cette valorisation du regard critique ("c'est vraiment un point important »...) est, d'après ce qui ressort des témoignages, liée au fait que les étudiants perçoivent les attentes de cette rubrique - exprimées en termes de précision, de profondeur, de rigueur et d'objectivation - comme caractéristiques des exigences du cadre universitaire, et de ce fait légitimes pour leur formation (voir aussi 3.1.3).

\begin{tabular}{|l|l|}
\hline upbE0 & $\begin{array}{l}\text { et est-ce que tu penses qu'il y a des exigences particulières pour arriver à faire ce travail- } \\
\text { là ? [...] }\end{array}$ \\
\hline \hline upbAJ1 & $\begin{array}{l}\text { un sens critique qui soit à la fois objectif, et en même temps pas indécis / donc il faudrait } \\
\text { que au niveau de l'exercice critique/ c'est important / ensuite c'est vrai que au niveau } \\
\text { simplement de l'écriture, des efforts de style, de présentation, de forme sont très } \\
\text { importants / et je pense que, un des prérequis, c'est vraiment la profondeur, aller le plus } \\
\text { loin possible et le plus précisément possible dans la visite / vraiment accorder beaucoup } \\
\text { d'importance aux détails // simplement y consacrer le temps nécessaire }\end{array}$ \\
\hline
\end{tabular}

42 Par ailleurs, le travail du « regard critique » dans le cadre du compte rendu est ressenti comme concourant notablement au développement d'une attitude nouvelle et d'un regard plus « professionnel » sur les faits culturels.

\begin{tabular}{|l|l|}
\hline upbLD1 & $\begin{array}{l}\text { pour ma part / le plus motivant c'était le regard critique / et euh / le deuxième, euh/ } \\
\text { enfin compte rendu regard critique / parce qu'on apprend à regarder des choses } \\
\text { auxquelles on prêtait pas attention / comme la scénographie / et c'est intéressant de voir } \\
\text { les liens / parce qu'avant on regardait un musée / bêtement et là / on a des automatismes } \\
\text { qui reviennent }\end{array}$
\end{tabular}

Ce qui pose question dans ce constat, c'est que l'on suppose en général que ce qui contribuerait le mieux à cette transformation du sujet et de son rapport à l'objet culturel serait à priori l'écriture réflexive, autrement dit la rubrique d'autoévaluation de ses apprentissages, en ce qu'elle permettrait à l'étudiant de prendre conscience de ses avancées. Or, dans les témoignages recueillis, l'écriture réflexive est l'objet d'incompréhension quant à son utilité et reléguée à une place secondaire, et c'est au contraire la rédaction plus scolaire d'une réflexion critique sur l'objet qui est ressentie comme le moteur de la transformation.

\subsubsection{Critères de validation et évaluation}

Les comptes rendus produits par les étudiants sont soumis à une évaluation individuelle critériée et commentée par les formateurs, à travers une grille d'évaluation présente sur chaque formulaire (grille non pondérée). Pour le premier compte rendu réalisé (sans valeur certificative), une note (sur 5) était attribuée au compte rendu par l'évaluateur ${ }^{7}$, en sus de la validation individuelle de chaque rubrique par des commentaires attestant (ou non) le respect des consignes fixées par la grille d'évaluation. Il nous a semblé intéressant de chercher à élucider les critères qui, au- 
delà des commentaires formatifs, guident l'évaluateur dans la validation des comptes rendus.

Nous avons cherché à étudier si certaines variables aisément mesurables pouvaient être corrélées avec la note reçue pour le compte rendu dans sa globalité. Nous avons ainsi calculé pour les rubriques «regard critique» ( $R C$ ) et "autoévaluation des apprentissages" ( $\mathrm{AE})$ : le nombre de mots, le nombre de fautes langagières (orthographe, syntaxe, ponctuation, sans pondération de la gravité des fautes) par 100 mots et le nombre de pronoms personnels de la première personne (singulier et pluriel confondus) par 100 mots, ces variables étant des indicateurs respectivement de la longueur (le développement) de la rédaction, du respect des normes linguistiques par l'étudiant et de la présence du scripteur dans son écriture. L'objectif était d'obtenir une rapide évaluation de la possible corrélation entre ces variables et la note reçue par le compte rendu.

Table 1. Coefficients de corrélation ( $r$ de Pearson) entre différentes variables textuelles et la note obtenue pour le premier compte rendu.

\begin{tabular}{|l|l|l|l|}
\hline Variables & Moyenne & Écart type & Éval. CR1 \\
\hline Évaluation du compte rendu 1 & 3,24 & 0,85 & 1,00 \\
\hline \hline Regard critique & & & \\
\hline Longueur & 210 & 93 & $0,31^{*}$ \\
\hline Présence du scripteur & 0,77 & 1,06 & $-0,17$ \\
\hline Autoévaluation des apprentissages & & & \\
\hline \hline Longueur & 190 & 97 & $0,42^{* *}$ \\
\hline Présence du scripteur & 3,92 & 2,16 & $-0,08$ \\
\hline \hline Respect de la norme ling. (invers.) & 3,26 & 2,18 & $-0,36^{*}$ \\
\hline
\end{tabular}

Note : ${ }^{*}<<0,05 * \star p<0,01(N=49)$

Les résultats montrent que la note obtenue est effectivement corrélée à la longueur des textes rédigés par les étudiants, et ce, de façon très significative dans le cas de la longueur de la rubrique "autoévaluation des apprentissages». Ceci n'est pas tant intéressant comme indicateur de ce qu'il faudrait faire pour être bien évalué, mais plutôt comme indicateur caractérisant les pratiques scripturales des étudiants répondant aux attentes de ce type de rédaction.

Par ailleurs, on observe également une corrélation significative entre le respect de la norme linguistique et la note obtenue. Par contre, la présence relative du scripteur dans son texte à travers l'utilisation de pronoms personnels autoréférentiels ne présente aucune corrélation avec les résultats de l'évaluation. Ceci tendrait à montrer, si du moins cela se voyait confirmé par d'autres recherches, que la pratique d'écriture réflexive d'un étudiant correspondant aux attentes des évaluateurs n'est pas 
caractérisée par une inscription explicite plus importante dans son texte. Une extrapolation de ces observations peut nous amener à mettre en question la corrélation entre des indices linguistiques discrets dans les productions écrites réflexives et la valorisation académique de ces productions et de leur auteur.

Nous avons d'autre part cherché à évaluer de façon plus globale la qualité des productions écrites pour les rubriques "regard critique " et "autoévaluation des apprentissages ». En regard avec ce que nous avions avancé précédemment (voir 3.2.1), la complexité de la rédaction de ces deux rubriques du compte rendu nous semble pouvoir être divisée en trois enjeux :

1. le développement d'un regard distancié et critique correspondant aux exigences universitaires (niveau d'approfondissement et de distanciation attendu) ;

2. l'appropriation de la posture adéquate (validante et objectivante pour le regard critique, autocentrée et heuristique pour l'autoévaluation des apprentissages) ;

3. la construction de savoirs pertinents (sur l'objet analysé pour le regard critique, sur soi pour l'autoévaluation des apprentissages).

En notant de façon élémentaire (sur une échelle à trois positions) et subjective (basée non sur des éléments aisément objectivables, mais plutôt sur notre expérience d'évaluation d'écrits académiques, appuyée par l'interprétation des commentaires des évaluateurs) la conformité des productions écrites de 10 étudiants avec ces trois dimensions de la rédaction critique et réflexive, nous avons cherché à valider leur pertinence pour l'évaluation de ce type d'écrit.

Table 2. Coefficients de corrélation ( $r$ de Pearson) entre l'évaluation des trois dimensions et la note obtenue pour le premier compte rendu.

\begin{tabular}{|l|l|l|l|}
\hline Variables & Moyenne & Écart type & Éval. CR1 \\
\hline \hline Regard critique & & & \\
\hline Niveau d'approfondissement/distanciation & 0,65 & 0,47 & $0,88^{* * *}$ \\
\hline \hline Appropriation de la posture adéquate & 0,70 & 0,35 & 0,54 \\
\hline \hline Construction de savoirs pertinents & 0,75 & 0,42 & 0,28 \\
\hline \hline Autoévaluation des apprentissages & & & \\
\hline \hline Niveau d'approfondissement/distanciation & 0,60 & 0,46 & $0,82^{* *}$ \\
\hline \hline Appropriation de la posture adéquate & 0,75 & 0,35 & $-0,19$ \\
\hline \hline Construction de savoirs pertinents & 0,60 & 0,46 & 0,53 \\
\hline
\end{tabular}

Note: ${ }^{* \star} p<0,01 * \star * p<0,001(N=10)$

Les coefficients de corrélation sont très clairs : on observe une corrélation très forte entre la notation du compte rendu et ce que nous avons évalué sous le concept de 
«niveau d'approfondissement de distanciation » dans la rédaction de l'étudiant. Ceci fait sens dans la mesure où nous avons nous-mêmes défini cet enjeu comme lié au développement d'un regard critique « correspondant aux exigences universitaires » : ce qui est le plus fermement sanctionné par les évaluateurs, c'est la superficialité, l'imprécision et le manque de rigueur. En cela, les intuitions des étudiants sur les exigences de l'université (voir 3.2.3) s'avèrent pertinentes.

51 Par contre, les deux autres dimensions que nous avions identifiées ne présentent aucune corrélation avec la note du compte rendu. Deux conclusions sont possibles : soit ces deux dimensions manquent de validité et de pertinence pour analyser les écrits critiques d'étudiants et devraient être abandonnées ou corrigées, soit le fait que l'étudiant s'approprie une posture énonciative adéquate (réflexive ou critique) et le fait qu'il ait identifié des savoirs pertinents à présenter dans sa rédaction n'ont en réalité pas une importance significative pour la validation des productions écrites des étudiants. Des recherches supplémentaires seront nécessaires pour infirmer ou confirmer l'une de ces interprétations.

\subsubsection{Liens avec la performance académique}

Enfin, nous avons cherché à savoir si la performance évaluée des étudiants dans le cadre de la réalisation du premier compte rendu pouvait être corrélée avec leur performance académique. Pour ce faire, nous avons calculé le coefficient de corrélation entre les notes du compte rendu et la moyenne académique (sur 20) des étudiants, ainsi qu'avec les points obtenus par les étudiants à l'examen d'un cours consacré à la maitrise du discours universitaire ${ }^{8}$, cours qui évalue directement les productions écrites des étudiants dans leur conformité aux attentes universitaires. Ces deux variables sont donc respectivement des indicateurs de la performance académique et de la performance discursive académique des étudiants.

Table 3. Coefficients de corrélation ( $r$ de Pearson) entre l'évaluation du premier compte rendu et les performances académiques.

\begin{tabular}{|l|l|l|l|l|}
\hline Variables & Moyenne & Écart type & Éval. CR1 & Perf. acad. \\
\hline \hline Évaluation du compte rendu 1 & 3,24 & 0,85 & 1,00 & \\
\hline \hline Performance académique & 11,18 & 2,75 & $0,38^{* *}$ & 1,00 \\
\hline Performance discursive (janvier) & 7,21 & 3,31 & $0,37^{* *}$ & $0,39^{* *}$ \\
\hline \hline Performance discursive (finale) & 10,33 & 3,34 & 0,28 & $0,54^{* * * *}$ \\
\hline
\end{tabular}

Note : ${ }^{\star *} p<0,01 * \star \star \star p<0,0001(N=49)$

Les résultats statistiques démontrent une corrélation forte entre l'évaluation du compte rendu et la performance académique globale, ainsi qu'avec la performance discursive académique lors de la première session (janvier) (corrélation non significative avec l'évaluation finale de la performance discursive). Ce dernier point s'explique sans doute par le fait que la rédaction du compte rendu arrive peu après la 
session de janvier et que les compétences d'écriture scientifique des étudiants sont à ce moment-là encore proches de celles évaluées en janvier.

\subsection{Perception des apports de l'ePortfolio}

Enfin, nous avons cherché à évaluer les apports externes attribués par les étudiants à l'expérience de l'ePortfolio, apports par rapport à l'ensemble de leur formation universitaire ou apports à titre personnel. L'objectif n'est pas ici d'évaluer quantitativement les conséquences du dispositif en termes d'apprentissage, mais bien d'analyser la perception qu'ont les étudiants de la valeur exogène de l'expérience. De ce fait, nous nous sommes basés exclusivement sur le contenu des entretiens.

Un certain nombre d'étudiants mentionnent d'abord des apprentissages en termes de connaissances, acquis à travers les activités réalisées qu'à la rédaction des comptes rendus. Nous ne les détaillerons pas ici, étant donné leur dimension moins globale. Il est cependant intéressant de noter que, lorsqu'ils sont interrogés sur l'apport que la participation au dispositif a pu avoir pour le reste de leur année de formation, les étudiants s'en tiennent pour la plupart à signaler ces connaissances apprises qui ont pu leur préparer l'accès à certaines matières d'autres cours.

Néanmoins, il serait erroné de penser que les étudiants ne perçoivent du dispositif que des apports en termes de connaissances, très localisés et occasionnellement mobilisables dans d'autres cours. Ils perçoivent manifestement une évolution dans leur façon d'aborder à la fois les objets culturels et leur projet d'études. Cette évolution se traduit, d'après les étudiants, par l'acquisition d'un regard différent sur l'art, sur les musées et expositions, etc. Un regard «plus professionnel » (upbHS1), plus «critique » (upbDS1).

\begin{tabular}{|l|l|}
\hline upbE0 & $\begin{array}{l}\text { ok // et est-ce que ça vous a semblé utile / [...] pour les autres cours ? premièrement / et } \\
\text { deuxièmement pour vous en général ? }\end{array}$ \\
\hline upbCL1 & euh ben c'est toujours utile / \\
\hline upbDS1 & $\begin{array}{l}\text { pour les autres cours je sais pas / mais pour nous surement / même si on se rend pas } \\
\text { toujours compte / il y a toujours un moment où on se dire "ah ben heureusement qu'on a } \\
\text { comme on se posait pas la question avant, ben ça nous apprend à avoir un meilleur regard } \\
\text { critique / et ça, on nous dit pourquoi en pratique scientifique, donc tous les travaux qu'on } \\
\text { rend, "justifiez tout", "critiquez ce que/ vos informations", etcaetera etcaetera / et euh je } \\
\text { suppose qu'aussi plus tard, on pourra pas se contenter de bêtement regarder les choses / } \\
\text { il faudra / il faudra donner un avis / dire si c'est bien, pas bien, pourquoi, etcaetera // } \\
\text { donc ça, je pense oui / mais pour les autres cours, pour le moment, ça n'a pas encore / il } \\
\text { n'y a pas encore eu trop de lien }\end{array}$ \\
\hline
\end{tabular}

Au-delà de ce nouveau regard sur les objets culturels, quelques étudiants font également état d'un changement survenu dans leur attitude vis-à-vis de leur projet d'étude. Ces transformations vont avant tout dans le sens d'une plus grande ouverture à l'ensemble de la formation, qui peut éventuellement conduire à refonder d'une façon plus solide son choix d'études et à mieux saisir le sens des différentes dimensions du 
programme universitaire. Le récit d'une étudiante l'illustre particulièrement bien : elle nourrissait un projet personnel d'orientation très précis, dicté par une passion d'enfance pour la paléontologie, ce qui l'avait donc conduit à s'inscrire au baccalauréat en histoire et de l'art et archéologie, pour sa composante en archéologie. Cependant, l'expérience de l'ePortfolio, en l'ouvrant à d'autres faits culturels et en l'amenant à porter sur ces faits un regard d'analyste, avait suscité chez elle un intérêt croissant pour les autres dimensions de sa formation. Elle était donc sortie d'une dynamique du projet ici relativement limitative par sa précision pour refonder son option d'orientation sur un intérêt large pour l'analyse de l'art et des civilisations.

Il est néanmoins difficile d'évaluer dans ces évolutions la part qui est à imputer à la rédaction des comptes rendus critiques et réflexifs, et celle qui est suscitée avant tout par la réalisation des activités culturelles. Ces aspects du dispositif sont bien évidemment liés, en ce compris dans leurs effets, mais il est en tout cas important de signaler que ces changements de perspective ne peuvent être attribués uniquement aux appels à la réflexivité dans les comptes rendus : les étudiants nous ayant signalé ces transformations n'en avaient pas pour autant témoigné dans leurs comptes rendus, et ne les associaient pas à l'exercice de l'autoévaluation des apprentissages.

Par ailleurs, lorsque nous interrogions les étudiants sur ce qu'ils avaient le sentiment d'avoir acquis d'utile pour eux et pour leur formation à travers l'expérience du portfolio, nous avons été interloqués par le fait que, de façon récurrente, les étudiants signalaient au premier abord le fait qu'ils avaient acquis des automatismes ou des habitudes d'analyse dans leurs activités, mais essentiellement utiles pour la réalisation des comptes rendus suivants! Sachant que les questions visaient plutôt les apprentissages méta et l'acquisition d'un nouveau regard, ces réponses faisant état d'une sorte de boucle autovalidante du dispositif nous ont parues relativement significatives, d'autant qu'elles sont apparues dans plus de la moitié des entretiens (et dans la plupart des autres entretiens où cela n'apparait pas, la question des acquis globaux de l'expérience n'avait pas été directement abordée). En voici deux exemples :

\begin{tabular}{|l|l|l|}
\hline upbE0 & $\begin{array}{l}\text { ce que tu retirais finalement comme savoir-être d'une visite, toi t'as l'impression que } \\
\text { surtout c'était comprendre l'attitude vis-à-vis d'un musée - je reformule ce que tu disais - } \\
\text { l'attitude à avoir vis-à-vis des oeuvres et du musée et savoir comment les appréhender? }\end{array}$ \\
\hline upbKM1 & $\begin{array}{l}\text { ben oui on apprend vraiment à critiquer / avec les moyens qu'on a pour l'instant je veux } \\
\text { dire / mais quand je rentre dans une expo, quand c'est pour faire un ePortfolio, } \\
\text { maintenant je sais exactement ce que je dois regarder euh / si ça c'est bien ou pas / } \\
\text { enfin... }\end{array}$ \\
\hline upbE0 & $\begin{array}{l}\text { et est-ce que le fait d'avoir fait du coup le compte rendu / enfin le compte rendu, le } \\
\text { regard critique etcétéra / vous a permis aussi de mieux comp/ enfin la fois où vous allez } \\
\text { juste après / d'avoir plus ces automatismes, ces regards différents? }\end{array}$ \\
\hline upbNH1 & oui ça devient / c'est... vraiment des automatismes \\
\hline upbLD1 & maintenant on sait vraiment / ce qu'il y a dans chaque rubrique \\
\hline
\end{tabular}

C'est une évidence : ce que le compte rendu enseigne le mieux, c'est à faire d'autres comptes rendus. Et de fait, en tant que forme écrite, il est aussi d'une certaine manière 
pertinent pour les étudiants d'apprendre à réaliser ce type de travail. Néanmoins, cette prépondérance de l'apprentissage de la forme canonique « compte rendu » - plutôt que d'une démarche critique, d'une attitude réflexive, etc. - qui ressort des entretiens ne peut être négligée. Nous reviendrons sur les implications de ces réponses plus loin (voir 4.3).

\section{Discussion et implications théoriques et pédagogiques}

\subsection{Autonomie et confrontation dans l'écriture réflexive}

61 Les entretiens individuels ont montré qu'à une exception près, tous les étudiants avaient rencontré des difficultés avec la rubrique d'autoévaluation de leurs apprentissages. Ces difficultés doivent certainement être pour partie mises sous le compte de la nouveauté de ce type d'écriture, mais il nous semble aussi que, bien que les coordinateurs du dispositif aient prévu un encadrement très important du portfolio, ils aient conçu la démarche réflexive comme profondément individuelle et autonome. L'étudiant est placé, seul, dans une activité (une visite de musée, l'assistance à un concert, etc.) et est censé être capable de prendre conscience - au moment de réaliser l'activité ou au moment de rédiger son compte rendu ? le dispositif ne le dit pas - des apprentissages qu'il a pu réaliser à cette occasion. Tant l'activité que la rédaction du compte rendu sont conçues comme totalement individuelles.

Si cette volonté d'autonomisation de l'étudiant fait pleinement sens dans une démarche comme celle qui est attendue par le retour descriptif et critique sur l'activité, elle pose problème au niveau de la mise en place d'une capacité réflexive. Elle laisse apparaitre en effet une conception de la réflexivité comme solitaire et autonome, conception qui, nous semble-t-il, est souvent présente dans les dispositifs visant à développer la réflexivité dans des formations non professionnalisantes. Or, cette conception est problématique à plus d'un titre.

Revenons aux sources du concept de réflexivité dans les pratiques éducatives, à savoir le modèle du "praticien réflexif» développé par Schön (1994). Selon ce modèle, la « réflexion-sur-l'action » menée par le professionnel dans le but de la réguler n'est pas réalisée sur le mode de l'introspection, comme si le professionnel se retirait après son action pour y réfléchir en solitaire: cette réflexion s'appuie sur tous les indices extérieurs reçus pour évaluer cette action. Il serait donc erroné d'imaginer le sujet réflexif comme un spécialiste de l'introspection. L'introspection seule présente en effet le risque de virer au nombrilisme et peut mener le sujet dans une voie inadéquate sans lui donner aucun repère lui permettant de corriger son erreur. La réflexivité doit donc être avant tout conçue comme une "altéro-réflexivité " (de Robillard, 2007, p. 135). Ceci s'applique à toute forme de réflexivité du sujet, qu'elle soit liée à une pratique professionnelle ou à une visée de développement personnel.

"Cette réverbération, cette réflexivité n'est pas réductible à la réflexion sur soi d'un je pense (cogito) pensant un objet (cogitatum) qui ne serait autre que lui-même. C'est l'image qui est renvoyée à un sujet connaissant par d'autres sujets connaissants équipés d'instruments d'analyse qui peuvent éventuellement leur être fournis par ce sujet connaissant » (Bourdieu, 2001, p15). 

de ses propres connaissances et un certain type de contenus « réflexifs » (notamment, évaluation de la modification des connaissances antérieures par l'activité). Bien sûr, ces consignes sont pertinentes et intéressantes, mais, face à des cas où l'étudiant fait plus librement état des réflexions que lui a inspirées l'activité, ces consignes fixent un cadre très strict de validité à l'attitude réflexive. Autrement dit, si l'étudiant fait preuve d'une certaine réflexivité, mais que celle-ci ne correspond pas aux attentes de l'exercice - en termes de distanciation et d'approfondissement, de contenus («savoirs» et « attitudes ») ou de posture (autocritique) -, elle sera jugée « hors sujet » et invalidée. Ce type d'évaluation fait sens dans le cadre de l'apprentissage d'une posture critique sur des objets culturels, comme celle qui apparait dans l'exercice du « regard critique », 
mais pose question pour un type d'écrit censé amener l'étudiant à développer petit à petit une capacité d'autoanalyse et d'autoexplicitation. La prépondérance et la rigidité de la consigne semblent ici particulièrement contreproductives.

En effet, dans ces cas d'invalidation de l'écrit réflexif, une contradiction se présente entre la contrainte de l'évaluation et la sincérité de la trace réflexive. L'élève fait face à une situation de " double contrainte ", autrement dit à des injonctions contradictoires : soit il cherche à apprendre pour lui-même, à exprimer ce qu'il a sincèrement ressenti ou appris au cours de son activité, mais qui pourrait décevoir l'évaluateur parce que différent du type d'explicitation que celui-ci attend, soit il cherche à satisfaire l'évaluateur et risque alors de construire un retour réflexif qui corresponde aux attentes perçues. Car le risque est bien celui-là : que l'étudiant ne prenne pas l'exercice du compte rendu comme un outil de développement pour lui, mais bien - et pourrait-on vraiment le lui reprocher, étant donné les enjeux présents? - comme un exercice de conformation parfaite aux attentes du format académique, dans laquelle faire apparaitre nuance, remise en question de soi et réflexivité est une stratégie bien évaluée.

On pourrait argüer du fait qu'il ne s'agirait là que d'un biais occasionnel de l'exercice réflexif. Cependant, il nous semble que cette contradiction est présente de manière généralisée dans ce type d'exercice. Vanhulle (2004, p. 29) écrit que, dans l'écriture réflexive, les étudiants " sont invités à produire non pas des "vérités vraies", mais à se mettre en recherche de validités diverses ». C'est aussi notre impression, dans la mesure où l'étudiant puise dans sa réflexion différentes réponses valides possibles, mais on observera qu'il n'y a alors plus ici de barrière qui contraindrait les étudiants à exprimer un ressenti propre ou une conclusion personnelle. Toute réponse valide, autrement dit toute réponse qui satisfera les attentes de l'évaluateur et du cadre (académique) sera bonne. Ceci peut paraitre évident, ou inévitable, mais pousse à questionner l'identité de cette "réflexivité » qui apparaitrait dans les écrits réflexifs formellement évalués, et à mettre sérieusement en doute la pertinence donc d'évaluer ce type d'écrit.

\subsection{2. Évaluer l'écrit réflexif}

71 Nous observons donc que l'évaluation peut entrainer des biais non négligeables, qui poussent l'étudiant à formater sa réponse, à la calquer sur l'idée qu'il se fait de la réponse idéale. Peut-on alors évaluer l'écriture réflexive?

72 Il nous semble tout d'abord qu'il faille, dans tous les cas, renoncer à toute évaluation certificative du produit de l'écriture réflexive, autrement évaluer la conformité des idées transmises par l'étudiant. Tout au plus, il est possible de certifier la réalisation du processus réflexif, mais encore faut-il pouvoir y avoir accès. En effet, la production écrite de l'étudiant n'est qu'une trace mise en forme de ce processus. Pour évaluer ce dernier, il faudra donc sans doute se baser sur d'autres sources d'information (par exemple, une vérification du fait que l'étudiant a bien réalisé les activités ou bien participé aux échanges qui étaient susceptibles d'entrainer la démarche réflexive), et on pourra très difficilement en évaluer la qualité (Slingeneyer et Bibauw, 2009, à paraitre).

73 Si l'on considère que la réflexivité est un objectif de formation à long terme, il est essentiel de considérer l'écriture réflexive comme un outil uniquement formatif, au 
service de l'étudiant. Hors d'un environnement formatif, la réflexivité est d'abord contreproductive : elle ne pousse pas à la réalisation immédiate des attentes, fait passer le sujet par des détours et des doutes... L'étudiant qui vise à réussir une évaluation n'aurait donc aucun intérêt à faire preuve de réflexivité. D'où l'importance de la cantonner au formatif.

Cette réserve émise, se pose encore la question de ce qui peut être évalué de façon formative. Vanhulle (2004, pp. 25-26) estime que « l'action langagière de l'étudiant, en tant qu'elle reflète une quête singulière de validité, ne peut pas s'évaluer pour ellemême ». Cependant, selon cette spécialiste du portfolio, il est possible d'évaluer «les contenus produits » : «il est bien attendu des étudiants qu'ils manient des concepts avec exactitude et créativité, qu'ils produisent des idées pertinentes [...], qu'ils osent des positionnements personnels, qu'ils sachent s'autoévaluer dans leur progression professionnelle» (25). Toutefois, nous serions tentés d'être plus stricts que Vanhulle sur ce qu'il convient d'évaluer. Il nous semble en effet que, même s'il est louable de vouloir mesurer à quel point ces étudiants mènent « loin » leur effort réflexif, la forme de cette évaluation dite "formative » est trop souvent celle d'un jugement de valeur, qui encouragerait de nouveau les étudiants à rechercher la désirabilité et la légitimité avant leur propre questionnement.

L'accompagnement de l'écriture réflexive par l'enseignant doit donc se faire sur un mode formatif stricto sensu. L'enseignant qui donne des retours sur la qualité même du processus devrait le faire en tant que formateur, voire en tant qu'accompagnateur de l'étudiant, et non sous une casquette d'évaluateur trop souvent confondue (par les apprenants en tout cas) avec celle d'un juge. Le journal dialogué que décrit Scheepers (2004) est ainsi un exemple remarquable de ce que peut être un accompagnement formatif qui échappe aux dynamiques évaluatives formatant l'écrit réflexif.

Ceci exige de mettre en place dans les dispositifs, et plus particulièrement dans les comptes rendus qui nous occupent, une distinction plus nette entre (1) la validation de l'engagement dans l'activité (dans le cas de l'ePortfolio, la vérification que l'activité extraacadémique a bien été menée avec le sérieux attendu'), (2) la validation de l'exploitation analytique et critique de l'activité, évaluée dans sa correspondance aux attentes académiques (en l'occurrence, les rubriques portant sur la description et le regard critique sur l'activité) et (3) le retour réflexif sur l'activité, qui ne serait pas évalué de façon formelle, voire pas évalué du tout, et qui, s'il donne lieu à des réactions du formateur, serait commenté sur un mode essentiellement coopératif. Ce n'est qu'alors que l'écriture réflexive pourrait être l'occasion d'un véritable dialogue entre étudiant et formateur, en évacuant les biais introduits par l'évaluation, et l'occasion surtout pour l'étudiant de prendre les rennes de son développement.

\subsection{Entre développement d'une démarche réflexive et apprentissage d'une nouvelle forme discursive}

Quel est l'apport global du dispositif d'ePortfolio pour l'apprentissage des étudiants? Les retours des étudiants sur celui-ci tendent à montrer qu'il présente une valeur globale très positive et qu'il apporte réellement une nouvelle dimension à la formation dans laquelle il s'insère. Il semble en particulier que le dispositif réussit parfaitement à exploiter de façon académique des activités réalisées hors du cadre des cours, en poussant les étudiants à approfondir leur expérience des objets culturels. Les comptes 
rendus attendus des activités ont manifestement un effet réel sur le développement chez l'étudiant d'une capacité d'analyse critique. Le dispositif d'ePortfolio remporte donc son pari de placer l'étudiant dans une dynamique d'autoformation qui puisse être à la source d'apprentissages réels.

Cependant, concernant la rubrique d'" autoévaluation des apprentissages » et l'objectif d'engager l'étudiant dans une démarche réflexive, le bilan nous parait plus mitigé. Par rapport à l'objectif de permettre "à l'étudiant de prendre conscience de ses forces et de ses faiblesses, de les exprimer et de proposer des pistes pour renforcer les premières et/ou compenser ou dépasser les secondes » (Druart et al., 2009, p. 23), il nous semble en effet que l'écriture réflexive appelée dans le compte rendu ne parvient pas en ellemême à jouer ce rôle.

Les perceptions des étudiants sur cette rubrique, et en particulier leur identification d'apports essentiellement autovalidants pour la rédaction des comptes rendus (voir 3.3), tendent à démontrer que cette forme d'écriture n'est pas reçue comme un lieu potentiel d'apprentissage, mais bien une sorte de "case à remplir ", de passage obligé dont on ne perçoit pas vraiment la valeur, et dont le remplissage s'effectue par l'application la plus stricte des consignes et la réalisation des attentes perçues des évaluateurs. Signalons surtout qu'aucun étudiant n'a réellement perçu cette rubrique à priori comme une occasion d'en apprendre plus sur lui ou de se questionner : si un apprentissage pouvait découler de sa rédaction, selon ces étudiants, ce n'était que sous forme d'effet induit, de conséquence nécessaire.

Dans ces conditions, l'écrit réflexif n'est pas perçu comme étant, en soi, une occasion d'autoformation, mais plutôt comme une nouvelle forme discursive imposée, que l'étudiant doit apprendre à maitriser. À l'extrême opposé d'un habitus réflexif (Perrenoud, 2001), ce que pousse alors à acquérir ce type d'écrit, ce sont alors des automatismes d'analyse et de rédaction, des formules toutes faites témoignant d'une remise en question de soi conforme aux attentes des formateurs. La " mise en scène » de la réflexivité (Rouxel, 2006) prend alors le dessus sur sa valeur formative, et conduit l'étudiant à construire de toutes pièces un récit de réflexivité, soit pour donner la preuve à l'enseignant qu'il est réflexif, soit pour se plier aux normes du genre, dont on ne peut évaluer ni la sincérité, ni l'impact sur les apprentissages.

81 Quelle est la source de ce dévoiement de l'écriture réflexive? Elle nous semble devoir être cherchée dans l'évaluation formelle et certificative de l'écrit réflexif, et dans le caractère trop autonome du dispositif réflexif, comme nous l'avons déjà dit, mais également dans des consignes trop restrictives pour cette rubrique d'autoévaluation des apprentissages. En effet, dans la mesure où les consignes de rédaction limitent cette autoévaluation au cadre strict de l'activité réalisée, et appellent à énoncer explicitement des savoirs précis et attitudes modifiées, cette écriture est difficilement pour l'étudiant le lieu d'une remise en question globale de sa place au sein de sa formation et de son rapport aux apprentissages. L'encadrement strict de l'écriture réflexive par des consignes trop précises amène ainsi les étudiants dans une position où ils ne "trouvent " pas ce qu'il « faudrait écrire ", ce qui témoigne clairement d'une mauvaise compréhension de la posture attendue dans ce type d'écrit.

Par ailleurs, on pourra se demander dans quelle mesure il n'est pas contreproductif de chercher à isoler la formation à la réflexivité en tant qu'activité propre. Les témoignages d'étudiants ont en effet fait part d'un certain développement réflexif issu 
plus globalement de l'ensemble du dispositif, sans qu'il soit pour autant réellement présent dans la rubrique qui appelait son explicitation.

\subsection{Des liens entre réflexivité et écriture}

83 Ces observations nous poussent à reconsidérer la valorisation des pratiques d'écriture réflexive comme étant, en soi, génératrices d'une démarche réflexive. Il nous semble que la mise en évidence du processus d'objectivation et de distanciation que génère l'écriture ( Albarello, 2004, p.109; Fayol, 1984) a conduit certains chercheurs à considérer trop rapidement que l'écriture induisait nécessairement une démarche réflexive chez le sujet scripteur (Chabanne et Bucheton, 2002). Nous souhaiterions nuancer cette idée.

Pour que l'écrit puisse être le vecteur d'une réflexivité du sujet, il faut d'abord que le sujet développe un certain rapport à l'écriture, qui sort des approches scolaires imposées, un rapport personnel et heuristique à l'outil (Bucheton, 2003). Or, "l'utilisation de l'écriture dans les études n'est que très rarement perçue comme un outil pour apprendre et réfléchir. [L'écrit] apparait peu comme un medium au service de la construction d'objets de savoir, encore moins comme un moyen de se développer, en tant que personne et professionnel en devenir " (Vanhulle et Deum, 2006, p. 12). Ceci nous semble être un premier écueil, non négligeable, qui implique qu'avant de pratiquer l'écriture réflexive dans un but réellement formatif, l'on se préoccupe du rapport à l'écriture des étudiants. Comme en témoignent Crinon et Guigue (2004, p. 169), «le passage par l'écrit n'est [...] pas une garantie que seront atteints des objectifs de construction de connaissances sur la pratique et de développement d'un rapport réflexif à l'exercice du métier. Il existe aussi des usages de l'écriture qui se bornent à fixer sur le papier des savoirs préexistants, dans une perspective de contrôle, sans mise en liens, sans réélaboration et sans souci de la précision que demande une communication à d'autres».

N'oublions pas que la réflexivité en tant que telle n'a pas lieu dans l'écriture : ce sont les moments d'arrêts ou les rythmes lents induits par la temporalité de l'écriture qui permettent à la pensée d'opérer une réflexion sur elle-même. Et, bien sûr, chaque scripteur n'occupera pas nécessairement ces pauses par des moments de remise en question réflexive. Autrement dit, l'écriture n'est pas tant génératrice que facilitatrice du processus réflexif. L'écriture vient plutôt étayer la démarche réflexive, c'est-à-dire lui servir de support pour se développer (Chabanne, 2006).

Il importe donc de ne pas surestimer le pouvoir de l'écriture dans la pratique réflexive et de ne pas se reposer que sur cette dimension écrite pour espérer voir l'apprenant développer une démarche autonome de réflexivité. Comme nous l'avons dit (voir 4.1), la réflexivité doit d'abord se construire dans la confrontation à l'altérité. L'écriture ne vient que dans un second temps, non comme exercice réflexif en tant que tel, mais comme effort d'intériorisation et de mise en évidence de ce qu'a enclenché la confrontation comme remise en question. 


\section{Conclusions}

\subsection{Limites de l'écriture réflexive encadrée}

87 À travers notre analyse du dispositif d'ePortfolio, nous avons signalé les différentes limites que rencontre l'exercice d'écriture réflexive, en l'occurrence la rédaction de l'« autoévaluation des apprentissages », dans le cadre de comptes rendus évalués dans un but certificatif. Ces limites nous semblent être transposables aux pratiques d'écriture réflexive formellement encadrées en général, c'est-à-dire imposées, dirigées par des consignes appelant de façon restrictive certains types de contenus et évaluées en référence à des normes externes de qualité.

Premièrement, l'écriture n'est pas automatiquement génératrice de réflexivité, mais plutôt un support pour celle-ci. Le dispositif d'écriture ne suffit donc pas à générer une démarche réflexive. Celle-ci doit se fonder sur la confrontation à l'altérité, soit par l'échange verbal avec l'autre, soit par la mise en confrontation d'un état de savoir avec une nouvelle réalité.

Deuxièmement, l'évaluation certificative de l'écriture réflexive - de même que toute forme d'évaluation qui, tout en se disant formative, vérifierait la conformité du produit réflexif à une norme externe de validité - parait entrainer de façon récurrente une double contrainte, qui tend à faire oublier à l'apprenant l'enjeu formatif de l'exercice pour se préoccuper prioritairement de se conformer aux attentes de l'évaluateur. En lien avec ce problème, la restriction des consignes de rédaction à une posture et à des contenus précis tend également à formater l'écriture réflexive et à la transformer en nouveau genre discursif à assimiler pour l'étudiant, plutôt qu'en outil d'autoapprentissage. C'est pourquoi il nous semble que l'écriture réflexive formellement encadrée - dans le double sens d'« encadrement normatif " par un évaluateur-juge et de "mise dans un cadre ", dans un format rigide, par des consignes fermées - n'est pas systématiquement vectrice d'un développement réflexif des sujets.

Ces considérations ne visent pas pour autant à discréditer le recours à l'écriture réflexive. Préparée et utilisée comme exercice à part, dans un environnement véritablement formatif où l'enseignant prend une posture d'accompagnateur, l'écriture réflexive peut jouer parfaitement son rôle, comme en témoignent différentes expériences ayant montré des résultats très encourageants (Terwagne, Vanhulle et Lafontaine, 2003 ; Scheepers, 2010). Pour cela, il faut toutefois donner à cette écriture un cadre propre, où les règles du jeu et les statuts des acteurs sont différents de ceux auxquels sont habitués les apprenants.

\subsection{Valeur pédagogique des comptes rendus d'activités extraacadémiques}

91 Cette mise en question de l'écriture réflexive ne doit pas non plus cacher l'importante valeur pédagogique du dispositif étudié et ses nombreux aspects positifs, soulignés à différentes reprises par les étudiants. Citons ici les éléments qui nous semblent les plus intéressants et les plus novateurs de l'ePortfolio, en envisageant les conditions de sa transposabilité. 
Il apparait que l'intérêt majeur de ce dispositif de portfolio et de comptes rendus réside dans son encouragement, sa valorisation et son exploitation d'activités réalisées hors du cadre de la formation. En l'occurrence, l'ePortfolio a permis de pousser les étudiants à devenir des analystes culturels actifs et critiques, en intégrant dans une formation universitaire la confrontation approfondie à des objets culturels institués. Le portfolio et le compte rendu d'activité semblent être ainsi des outils précieux pour les formateurs cherchant à insérer dans un curriculum plus théorique un rapport à des objets hors cadre. Il nous semble que cette réussite peut ainsi être associée à l'efficacité d'autres pratiques d'écriture réflexive exploitant des activités réalisées par les apprenants hors du cadre scolaire, comme c'est le cas des écrits réflexifs touchant aux expériences de lecture (Terwagne et al., 2003 ; Ledur et De Croix, 2005).

D'autre part, le dispositif réussit le pari de l'autoformation des étudiants, en mettant en place une structure d'apprentissage complexe et un suivi individuel conséquent, qui permettent aux étudiants de tirer pleinement parti d'un rapport différent aux objets étudiés.

Enfin, les comptes rendus d'activités, comme le montrent les retours des étudiants sur ceux-ci, se révèlent tout à fait efficaces pour aider les étudiants à acquérir et à développer peu à peu une posture analytique, critique et approfondie, face aux contenus de leurs études. L'exercice du « regard critique » semble en effet bien compris et bien assumé par les étudiants.

Il ressort de cette divergence de résultat entre ces deux parties des comptes rendus, consacrées respectivement à l'approche critique et au retour réflexif, que l'apprentissage d'une attitude critique vis-à-vis d'objets extérieurs à soi doit être réalisé selon des modalités bien distinctes d'un dispositif visant l'acquisition d'une attitude réflexive sur soi et sur son apprentissage. Les enseignants qui cherchent à former des sujets réflexifs et critiques devront donc bien dissocier ces deux objectifs, en mettant en place des outils, des pratiques d'écriture et des modalités d'évaluation propres à chaque dimension du développement de l'apprenant.

Nous tenons à remercier chaleureusement pour leur encadrement et leurs conseils Jean-Louis Dufays, Léopold Paquay, Marc Romainville et Francine Thyrion, ainsi que pour sa précieuse collaboration Marc Slingeneyer. Par ailleurs, cet article n'aurait pu advenir sans les encouragements et les suggestions toujours fines et perspicaces de Sébastien Marlair. Nous lui adressons tous nos remerciements.

\section{BIBLIOGRAPHIE}

Albarello, L. (2004). Devenir praticien-chercheur. Comment réconcilier la recherche et la pratique sociale. Bruxelles : De Boeck.

Barney, G. G. et Strauss, A. L. (1967). The Discovery of Grounded Theory : Strategies for Qualitative Research. Chicago : Adline. 
Bibauw, S. et Dufays, J.-L. (à paraitre). Les pratiques d'écriture réflexive en contexte de formation générale. Repères pédagogiques, 2 .

Bishop, M.-F. et Doquet-Lacoste, C. (2008). Place et statut du récit dans les "écrits intermédiaires" à l'école : carnets de lecture, carnets d'expériences et d'observations. Pratiques, 133-134, 165-191.

Bourdieu, P. (2001). Science de la science et réflexivité. Paris : Raisons d'agir.

Bucheton, D. (2003). Du portfolio au dossier professionnel : éléments de réflexion. Tréma, 20-21, 43-54.

Cerisier, J.-F. (2006). Le portfolio d'élève : pour qui et pour quoi ? Médialog, 57, 38-41.

Chabanne, J.-C. (2006). Écriture réflexive, construction de la pensée et des connaissances chez les élèves d'école primaire. Dans M. Molinié et M.-F. Bishop (Éds.), Autobiographie et réflexivité (pp. 51-68). Cergy-Pontoise : Université de Cergy-Pontoise.

Chabanne, J.-C. et Bucheton, D. (2002). Introduction. Dans J.-C. Chabanne et D. Bucheton (Éds.), Parler et écrire pour penser, apprendre et se construire. L'écrit et l'oral réflexifs (pp. 1-23). Paris : PUF.

Crahay, M. et Marlair, S. (2008, 24-26 avril). Les conditions énonciatives du discours universitaire dans son rapport au savoir. Communication présentée au colloque "Les discours universitaires : formes, pratiques, mutations", Bruxelles.

Crinon, J. (2002). Écrire le journal de ses apprentissages. Dans J.-C. Chabanne et D. Bucheton (Éds.), Parler et écrire pour penser, apprendre et se construire. L'écrit et l'oral réflexifs (pp. 123-143). Paris : PUF.

Crinon, J. et Guigue, M. (2002). Être sujet de son écriture : une analyse de mémoires professionnels. Spirale, 29, 201-219.

Crinon, J. et Guigue, M. (2004). Écrire ses pratiques dans des mémoires professionnels. Dans C. Blanchard-Laville et D. Fablet (Éds.), Écrire les pratiques professionnelles. Dispositifs d'analyse de pratiques et écriture (pp. 167-198). Paris : L'Harmattan.

de Robillard, D. (2007). La linguistique autrement : altérité, expérienciation, réflexivité, constructivisme, multiversalité : en attendant que le Titanic ne coule pas. Carnets d'atelier de sociolinguistique, 1, 81-228.

Druart, E., Verslype, L. et Corten-Gualtieri, P. (2009). Valoriser le parcours extra-académique de l'étudiant. Un ePortfolio pour valoriser les acquis extra-académiques des étudiants en baccalauréat d'archéologie et histoire de l'art. Éducation - Formation, e-291, 21-30.

Dufays, J.-L. (2004). Le rapport de stage : quelle évaluation pour quels enjeux ? Dans J.-L. Dufays et F. Thyrion (Éds.), Réflexivité et écriture dans la formation des enseignants (pp. 111-134). Louvainla-Neuve : Presses universitaires de Louvain.

Dufays, J.-L. et Thyrion, F. (Éds.). (2004). Réflexivité et écriture dans la formation des enseignants. Louvain-la-Neuve : Presses universitaires de Louvain.

Fayol, M. (1984). La distanciation dans le langage : l'exemple du calcul de l'origine dans le récit écrit d'expérience personnelle chez l'enfant de 6 à 10 ans. Paris : PUF.

Gauthier, P.-D. (2008, 5-7 mai). Typologie, processus réflexifs et usages du portfolio numérique. Communication présentée au colloque "ePortfolio \& Digital Identity", Montréal.

Girardeau, B. et Huver, E. (2009). Le biographique et la formation des enseignants de langues : vers l'émergence d'une posture de praticien réflexif ? Carnets d'atelier de sociolinguistique, 4. 
Kaufman, J.-C. (2004). L'entretien compréhensif. Paris : Armand Colin.

Ledur, D. et De Croix, S. (2005). Écrire son autobiographie de lecteur ou comment entrer en didactique de la lecture. Nouveaux cahiers de la recherche en éducation, 8 (1), 31-40.

Paquay, L. et Sirota, R. (Éds.) (2001). Le praticien réflexif, la diffusion d'un modèle de formation. Recherche et formation, 36 .

Perrenoud, P. (2001). De la pratique réflexive au travail sur l'habitus. Recherche et formation, 36, 131-162.

Ricard-Fersing, É., Dubant-Birglin, M.-J. et Crinon, J. (2002). Mémoires professionnels et portfolios dans la formation des enseignants. Une étude comparative. Revue française de pédagogie, 139, 121-129.

Romainville, M. (2007). Conscience, métacognition, apprentissage : le cas des compétences méthodologiques. Dans F. Pons et P.-A. Doudin (Éds.), La conscience chez l'enfant et chez l'élève (pp. 108-130). Québec : Presses de l'Université du Québec.

Rouxel, A. (2006). Genres biographiques et genres de la réflexivité. Dans M. Molinié et M.-F. Bishop (Éds.), Autobiographie et réflexivité (pp. 15-30). Cergy-Pontoise : Université de CergyPontoise.

Scheepers, C. (2004, 24-26 août). Le journal de formation : un écrit heuristique à double Je. Actes du 9e colloque de l'AIRDF, Québec.

Scheepers, C. (2005). Comment évaluer la réflexivité ? Communication présentée au 18e colloque international de l'ADMEE-Europe, Reims.

Scheepers, C. (2006). L'écriture réflexive, entrée dans la culture. Le français dans le monde / Recherches et applications, 39, 96-106.

Scheepers, C. (2010). Le travail de fin d'études, un discours en quête d'auteur. Pratiques langagières réflexives en formation initiale d'enseignants. Thèse de doctorat, Université de Liège / Université Paris VIII, Liège / Paris.

Schön, D. A. (1994). Le praticien réflexif. À la recherche du savoir caché dans l'agir professionnel (D. Gagnon et J. Heynemand, Trad.). Montréal : Logiques.

Slingeneyer, M. et Bibauw, S. (à paraitre). Des pratiques réflexives au 3e degré du secondaire : pourquoi? comment? Enjeux, 77.

Slingeneyer, M. et Bibauw, S. (2009). Développer la réflexivité par l'écrit en fin de secondaire. Échanges, 19, 8-15.

Terwagne, S., Vanhulle, S. et Lafontaine, A. (2003). Les cercles de lecture. Interagir pour développer ensemble des compétences de lecteurs. Bruxelles : De Boeck.

Vanhulle, S. (2004). L'écriture réflexive, une inlassable transformation sociale de soi. Repères, 30, 13-31.

Vanhulle, S. (2006). Genre réflexif, portfolio et didactique des savoirs professionnels. Dans A. Akkari et S. Heer (Éds.), La pédagogie de l'alternance dans la formation des enseignants (pp. 23-36). Porrentruy : HEP BEJUNE.

Vanhulle, S. et Deum, M. (2006). L'écriture réflexive en formation initiale d'enseignants : entre réconciliation avec l'écrit et apprentissage de la rigueur conceptuelle. Langage et pratiques, 37, 6-18. 
Viau, R. et Bouchard, J. (2000). Validation d'un modèle de dynamique motivationnelle auprès d'élèves du secondaire. Revue canadienne de l'éducation, 25(1), 16-26.

Vygotski, L. S. (1997). Pensée et langage (F. Sève, Trad.). Paris : La dispute.

\section{NOTES}

1. Projet FRFC-pédagogie universitaire $n^{\circ} 2.4588 .08$ " L'écriture réflexive au service de la qualité des apprentissages. Quelles pratiques effectives à la fin du secondaire et à l'entrée à l'université, et quel impact sur la motivation et le travail des étudiants? ", mené à l'Université catholique de Louvain (Belgique) en 2008 et 2009, sous la direction de J.-L. Dufays, L. Paquay, M. Romainville et Fr. Thyrion.

2. Il est intéressant de noter que les chercheurs qui étudient les pratiques réflexives sont en général de deux types: soit il s'agit d'enseignants-chercheurs présentant une pratique particulière qu'ils ont conçue dans le cadre de leur enseignement avec un intérêt pédagogique pour la réflexivité, soit il s'agit de chercheurs en sciences de l'éducation qui évoquent la réflexivité comme une dimension des pratiques enseignantes existantes ou potentielles dimension à développer, à jauger, à enseigner ... -, mais sans chercher à étudier les pratiques effectives en termes de réflexivité.

3. Ce dispositif de formation a été conçu à l'Université catholique de Louvain par une équipe d'enseignants et d'assistants, appuyés par des représentants des étudiants et des conseillers pédagogiques. Il a en particulier été mis en place et coordonné par Laurent Verslype et Emmanuelle Druart. Sa conception a été initiée dans le cadre d'un projet du Fonds de développement pédagogique de l'UCL. Il apparait dans le programme de cours sous le titre « Projets individuels et parcours extra-académique ».

4. Il est à noter que, après un bilan global sur le dispositif, réalisé au terme de la première année de sa mise en place, les intitulés et les consignes des formulaires ont été partiellement revus ou amplifiés en octobre 2009, entre autres pour mieux encadrer le travail des étudiants. Par souci de cohérence avec nos données, nous décrirons néanmoins les consignes reçues par les étudiants que nous avons observés.

5. N'ont donc pas été pris en compte une vingtaine d'étudiants n'ayant pas présenté tous les examens finaux, soit du fait de réorientations en cours d'année, soit du fait d'abandons de leurs études. Bien que l'étude des impacts de l'écriture réflexive sur ces étudiants en situation de désengagement ou de réorientation présenterait beaucoup d'intérêt, ce n'était pas possible dans notre cadre de recherche, du fait que ces étudiants n'ont en général pas participé de façon notable à l'ePortfolio (qui commençait au deuxième semestre).

6. Dans la première version du questionnaire, la rubrique demandait à l'étudiant d'évaluer ses apprentissages en termes de "savoirs" et de "savoir-être ». Ce dernier terme était à lui seul aussi sans doute responsable de certaines incompréhensions, même s'il était précisé dans les consignes en "attitudes et valeurs". La version mise à jour du questionnaire a maintenant abandonné ce terme pour le substituer par celui d' «attitudes».

7. Pour les comptes rendus suivants, une note similaire est également attribuée, mais seulement après l'entretien individuel entre l'étudiant et son formateur, ratifiant la réalisation des comptes rendus de l'année. Ces notes n'étaient pas accessibles au moment du recueil de nos données.

8. Cours "Pratiques du français à l'université ", coordonné par Fr. Thyrion, en 2008-2009 à l'Université catholique de Louvain.

9. Cette validation de l'engagement dans l'activité est déjà présente dans les comptes rendus étudiés, l'étudiant devant apporter différentes preuves ou traces physiques de son activité. Même si cette validation est alors très sommaire, il nous semble que cela peut être tout à fait suffisant. Il 
importera cependant de vérifier si ce type de validation de l'engagement est adéquat au regard des objectifs fixés au dispositif.

\section{RÉSUMÉS}

Cet article vise à interroger les pratiques d'écriture réflexive encadrée (imposée et évaluée) dans le cadre de la formation générale d'étudiants. Par l'analyse d'un dispositif de portfolio électronique pour lequel des étudiants de première année d'université doivent réaliser des comptes rendus d'activités extraacadémiques, nous cherchons à étudier quel a été leur mise en œuvre et leur perception des exercices appelant à la rédaction critique et à l'écriture réflexive. Les résultats des enquêtes tendent à faire apparaitre un certain nombre de limites de la portée de l'écriture réflexive, lorsqu'elle est normativement encadrée, imposée et évaluée. Le compte rendu réflexif montre néanmoins une valeur non négligeable pour l'exploitation approfondie et critique d'activités réalisées hors du cadre de formation.

This paper addresses the issue of, supervised reflective writing in students' general education. Through analyzing an electronic portfolio device where first year college students have to write reflective reports on extracurricular activities, we study how they carried out and perceived reflective and critical writing. Results tend to show some limits in reflective writing, when it is strictly supervised, compulsory and assessed. Nonetheless, the reflective report retains a significant value when fostering critical and in-depth use of student activities performed out of formal settings.

\section{INDEX}

Mots-clés : écriture réflexive, réflexivité, formation en autonomie, compte rendu, portfolio d'apprenant, portfolio électronique, entrée à l'université, évaluation

Keywords : reflexive writing, reflexive learning, activity report, learning portfolio, electronic portfolio, assessment

\section{AUTEUR}

\section{SERGE BIBAUW}

\section{CRIPEDIS/IACCHOS}

Université catholique de Louvain

Louvain-la-Neuve, Belgique 